\title{
La recirculación del agua residual industrial en el costo de reúso del proceso
}

\author{
The recirculation of industrial wastewater in the cost of reuse of the process \\ Recirculação de águas residuais industriais ao custo da reutilização do proceso
}

Pedro Córdova Mendoza

pedro.cordova@unica.edu.pe

https://orcid.org/0000-0003-3612-1149

Universidad Nacional San Luis Gonzaga, Ica-Perú

\author{
Isis Cristel Córdova Barrios \\ isis.cordova@unica.edu.pe
}

https://orcid.org/0000-0002-3569-2671

Universidad Nacional San Luis Gonzaga, Ica-Perú Universidad Autónoma de Ica, Chincha Alta-Perú

\author{
Teresa Oriele Barrios Mendoza \\ oriele.barrios@unica.edu.pe \\ https://orcid.org/0000-0002-6466-7766 \\ Universidad Nacional San Luis Gonzaga, Ica-Perú
}

\section{RESUMEN}

El objetivo de la investigación fue evaluar la recirculación del agua residual industrial y su influencia en el costo de reúso del proceso. Material y métodos la investigación realizada fue de tipo experimental, nivel descriptivoexplicativo, investigación aplicada. Resultados la norma DS N003-2002PRODUCE, establece los Límites Máximos Permisibles y Valores Referenciales para la actividad industrial de curtiembre con un $\mathrm{pH}$ entre $6-9$, temperatura $35^{\circ} \mathrm{C}$, se dispuso la herramienta MATLAB, que recoge toma de pruebas del proceso de la curtiembre, como lo indica las coordenadas UTM. Los costos totales (S/d): 1945.36 a 1870.98 , dependiendo del reciclo del agua residual (X1) de 0.6 a 0.75 , la carga de retiro de la planta de tratamiento (X2): $33.22 \mathrm{Kg} / \mathrm{h}$ a $39.41 \mathrm{Kr} / \mathrm{h}$ y la carga de retiro en la descarga a la fuente (X3): $19.72 \mathrm{Kg} / \mathrm{h}$ a $10.57 \mathrm{Kg} / \mathrm{h}$. Discusión se evidenciaron que los tratamientos $\mathrm{X} 1, \mathrm{X} 2$, $X 1 X 2$, influenciaron en los costos de reúso del proceso. Conclusión el análisis del costo de reúso del proceso depende del porcentaje de reciclo del agua residual de la planta de tratamiento, del porcentaje de retiro en la planta de tratamiento y de ambos tratamientos como el reciclo y del porcentaje de retiro en la planta de tratamiento.

Palabras clave: Agua de suministro; Reúso de agua industrial; Tratamiento de aguas residuales

\section{ABSTRACT}

The objective of the research was to evaluate the recirculation of industrial wastewater and its influence on the reuse cost of the process. Material and methods The research was of the experimental type, descriptive-explanatory level, applied research. Results the DS $\mathrm{N}^{\circ} 003-2002$ PRODUCE norm, establishes the Maximum Permissible Limits and Reference Values for the tannery industrial activity with a $\mathrm{pH}$ between $6-9$, temperature $35^{\circ} \mathrm{C}$, the MATLAB tool was used, which collects tests of the tannery process, as indicated by the UTM coordinates. The total costs (S/d): 1945.36 to 1870.98 , depending on the wastewater recycling $(X 1)$ from 0.6 to 0.75 , the withdrawal load of the treatment plant (X2): $33.22 \mathrm{Kg} / \mathrm{h}$ to $39.41 \mathrm{Kr} / \mathrm{h}$ and the withdrawal load in the discharge to the source $(X 3): 19.72 \mathrm{Kg} / \mathrm{h}$ to $10.57 \mathrm{Kg} / \mathrm{h}$. Discussion it was evidenced that the treatments $X 1, X 2, X 1 X 2$, influenced the reuse costs of the process. Conclusion the analysis of the reuse cost of the process depends on the percentage of recycling of the wastewater from the treatment plant, the percentage of withdrawal in the treatment plant and both treatments as the recycling and the percentage of withdrawal in the treatment plant.

Key words: Water supply; Industrial water reuse; Wastewater treatment

\section{RESUMO}

O objetivo da pesquisa era avaliar a recirculação das águas residuais industriais e sua influência no custo de reutilização do processo. Material e métodos A pesquisa realizada foi do tipo experimental, de nível descritivoexplicativo, pesquisa aplicada. Resulta a norma DS N003-2002-PRODUCE, estabelece os Limites Máximos Permitidos e Valores de Referência para a atividade industrial de curtume com um $\mathrm{pH}$ entre $6-9$, temperatura $35^{\circ} \mathrm{C}$, foi utilizada a ferramenta MATLAB, que coleta testes do processo de curtume, como indicado pelas coordenadas UTM. Os custos totais (S/d): 1945,36 a 1870,98 , dependendo da reciclagem das águas residuais $(X 1)$ de 0,6 a 0,75, da carga de retirada da estação de tratamento $(\mathrm{X} 2)$ : $33,22 \mathrm{Kg} / \mathrm{h}$ a $39,41 \mathrm{Kr} / \mathrm{h}$ e da carga de retirada na descarga até a fonte $(X 3)$ : $19,72 \mathrm{Kg} / \mathrm{h}$ a $10,57 \mathrm{Kg} / \mathrm{h}$. A discussão ficou evidente que os tratamentos $\mathrm{X} 1, \mathrm{X} 2, \mathrm{X} 1 \mathrm{X} 2$ influenciaram os custos de reutilização do processo. Conclusão A análise do custo de reutilização do processo depende da taxa de reciclagem das águas residuais da estação de tratamento, da taxa de retirada na estação de tratamento e de ambos os tratamentos, tais como reciclagem e taxa de retirada na estação de tratamento.

Palavras-chave: Abastecimento de água; Reuso de água industrial; Tratamento de águas residuais; Tratamento de águas residuais 


\section{INTRODUCCIÓN}

El siglo XXI, exige mayor compromiso y con responsabilidad en la sostenibilidad de la calidad del ambiente que establece un requerimiento esencial para la salud humana y el desarrollo sostenible (1). A finales del siglo XVIII con la Revolución Industrial marca un punto de inflexión en la historia, modificando e influenciando todos los aspectos de la vida cotidiana de una u otra manera, esto ha permitido que han aumentado las demandas sobre los recursos hídricos en el planeta. En la actualidad se sostiene que el ambiente y el crecimiento económico es un producto social, es decir, el resultado de la acción humana sobre el medio natural. Esta realidad plantea, los 17 Objetivos de Desarrollo Sostenible (ODS) definidos por Naciones Unidas que abordan los mayores retos de la humanidad desde una perspectiva global y transversal, con una visión a futuro hacia el año 2030. Los nuevos paradigmas están cambiando las nociones sobre qué son estos recursos y cómo deben utilizarse, de hecho, el aumento del uso del agua ha impulsado la búsqueda de fuentes alternativas como la desalinización del agua de mar y el reúso de aguas residuales tratadas (2).

En el mundo la industria de procesamiento en húmedo de textiles, es una de las industrias con mayor uso de agua y productos químicos, incluye procesos de teñido, acabadoe impresión (3). La utilización de más de 3600 tintes y 8000 productos químicos diferentes se informó en la literatura (4), (5). El procesamiento húmedo de textiles genera aguas residuales con una gran cantidad de colorantes y productos químicos usados que pueden tener impactos negativos en el medio ambiente acuático y la vida (6), (7).

La Autoridad Nacional del Agua (ANA), a través del Consejo de Cuenca, autoriza el reúso del agua residual tratada, según el fin para el que se destine la misma, en coordinación con la autoridad sectorial competente y, cuando corresponda, con la Autoridad Ambiental Nacional (8), artículo $82^{\circ}$, reutilización de agua residual.

La escasez de aguas naturales aprovechables es cada vez mayor, debido al crecimiento demográfico, la urbanización y el cambio climático. La situación ha generado la necesidad del aprovechamiento de las aguas residuales tratadas a nivel nacional e internacional para la agricultura, áreas verdes, cultivos forestales, riego de caminos y vías de acceso, entre otros tipos de tratamiento de las aguas industriales, como es el caso de los procesos de reciclaje para el reúso de aguas residuales en la industria (9).

\section{Bases teóricas}

Reutilización de agua residual, la escasez de agua y la precariedad del tratamiento de las aguas residuales municipales, asociada a la alta vulnerabilidad social y la escasez de recursos en las regiones semiáridas, agravan las disparidades sociales y hacen imposible mejorar la calidad de vida de la población (10). La reutilización del agua comenzó a aumentar tanto en el número de proyectos como en los tipos de aplicaciones de agua reciclada a medida que el tratamiento de aguas residuales, 
los procesos de desinfección y las técnicas analíticas físicas químicas y microbiológicas se volvieron más sofisticadas durante la primera mitad del siglo XX. De manera similar, los estándares de reutilización del agua evolucionaron para regular el uso de agua reciclada para riego. Durante este tiempo, los recursos hídricos en general fueron adecuados para satisfacer todas las necesidades de agua potable y no potable, y el uso de agua residual tratada a menudo se basó en la oportunidad, la conveniencia y la economía (11).

\section{Costo de reúso del proceso}

La revisión de la literatura académica sobre el diseño y evaluación de tarifas relacionadas con los servicios de agua, una de las conclusiones fue que en las próximas décadas el diseño de sistemas tarifarios óptimos será cada vez más importante a la hora de buscar mecanismos para financiar los servicios de agua (12).

En la industria, en general, uno de los principales objetivos es el ahorro en el consumo de agua (13), idealmente sería lo mejor reciclar toda el agua que se va utilizando, lo que evitaría el gasto por el consumo de agua de suministro a la planta, por tal razón, para obtener el costo total de reúso del proceso del agua residual, se deben tomar en cuenta el costo de tratamiento de agua al reciclo, costo de tratamiento de agua de descarga y el costo del agua de abastecimiento, ecuación 1.

Costo Total $\left(\frac{S / \cdot}{d}\right)=$ Costo agua de abastecimiento $\left(\frac{S / \cdot}{d}\right)+$ Costo tratamiento agua al reciclo $\left(\frac{S / \cdot}{d}\right)+$
Costo tratamiento agua de descarga $\left(\frac{S / \cdot}{d}\right)$

(Ecuavión 1)

Sistema integrado de reciclo de aguas residuales industriales, el reciclo integrado de aguas residuales industriales, se ha convertido en un tema importante en los últimos años, especialmente en los países en desarrollo, $y$ este se puede obtener al comprender tres aspectos del sistema de recolección, los procesos de tratamiento y el objetivo de reutilización, denominados conjuntamente "grupo funcional" (14).

El objetivo de la investigación fue evaluar la recirculación del agua residual industrial y su influencia en el costo de reúso del proceso, como consecuencia del estudio de investigación, que al aplicar el análisis estadístico en base monitoreo realizado in situ en las fechas programadas en coordinación con la empresa, se hizo necesario la aplicación de software de procesamiento lógico MATLAB, registrándose el algoritmo de costo de reúso del proceso, se accedió a los análisis respectivos para obtener los parámetros del estudio para obtener la simulación del costo de reúso del proceso (15). En el estudio propuesto se ha considerado el software Matlab que posee herramientas especiales, utilizándose el lenguaje de programación en la modelación del costo de reúso del proceso del agua residual en la empresa curtiduría. 


\section{MATERIALES Y MÉTODOS}

El departamento de Ica es uno de los veinticuatro departamentos que forman la República del Perú, está ubicado en el centro oeste del país, limitando al norte con Lima, al este con Huancavelica y Ayacucho, al sur con Arequipa y al oeste con el Océano Pacifico (16), con $2132783 \mathrm{~km}^{2}$ es el sexto departamento menos extenso y se fundó el 30 de enero de 1866, su territorio es casi por completo parte del desierto costero del Perú y conforma el llamado gran tablazo de Ica, con sus cinco provincias y es como sigue: Chincha, Pisco, Ica, Palpa y Nasca. La Provincia de Pisco tiene una superficie total de $397819 \mathrm{~km}^{2}$, donde destaca su amplia producción agroexportadora y empresas industriales de transformación de la metería prima, que determinan una región en pleno desarrollo industrial.

\section{Puntos de monitoreo en el proceso de la calidad de agua residual}

Se establecieron tres puntos de monitoreo en el proceso de recirculación del agua residual industrial con fines de costo de reúso del proceso en la empresa curtiduría, con las siguientes coordenadas UTM. El punto E-1 (8 $483851 \mathrm{~N}-371848 \mathrm{E})$, el punto E-2 (8 483755N - $371748 \mathrm{E})$ y el punto E-3 (8 $483762 \mathrm{~N}-371$ 843E), (Coordenadas UTM: Datum WGS84 Huso 18 Sur), la altitud media a 17 m.s.n.m. (Figura 1).

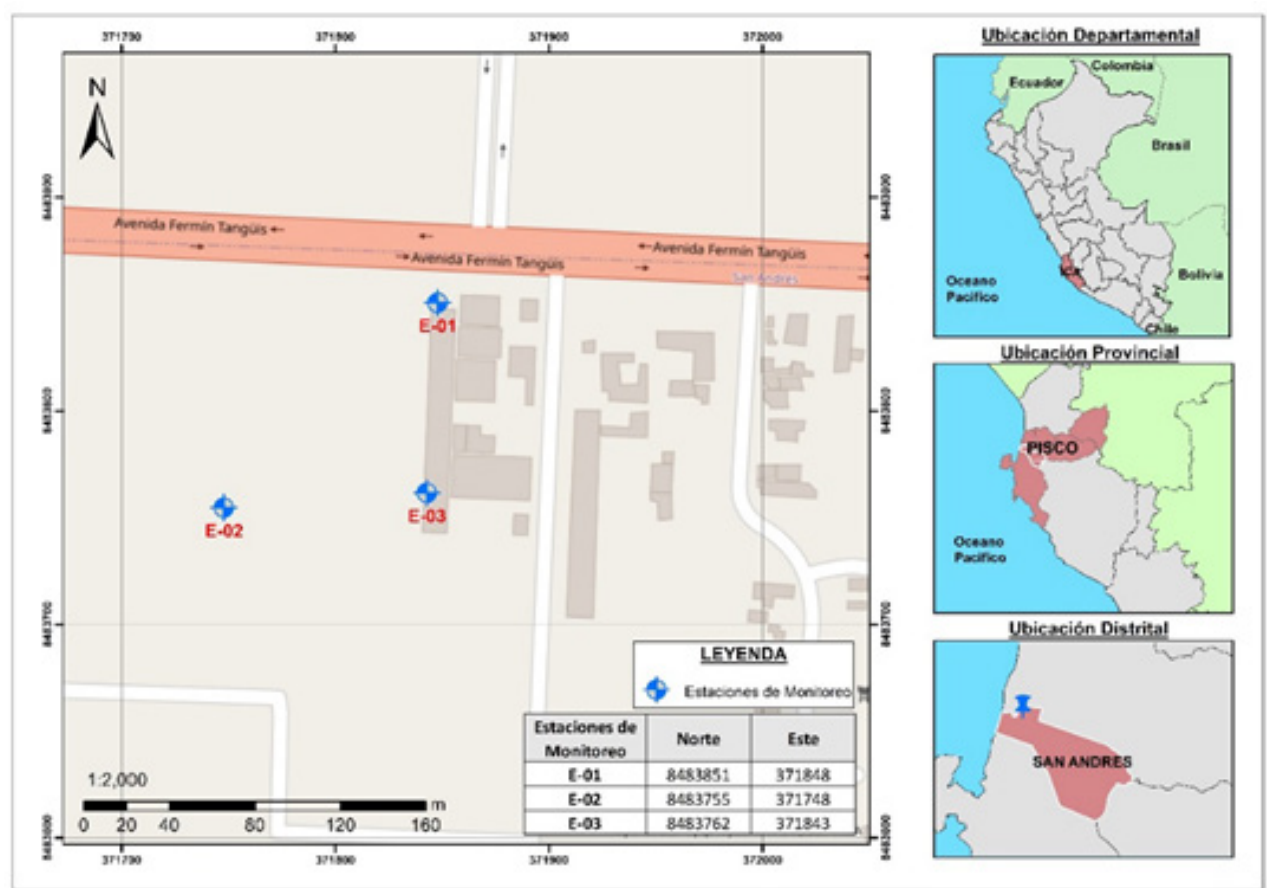

Figura 1. CLocalización de los puntos de monitoreo en el proceso de recirculación del agua residual industrial - Cercado de la Provincia de Pisco, donde se localizaron los puntos para el monitoreo: E-01, E-02 y E-03. 
Se realizaron tres pruebas en la empresa curtiduría, ubicado en el cercado de la Provincia de Pisco, Región de Ica. La investigación realizada fue de tipo experimental, con los siguientes criterios metodológicos considerados: prospectivo las pruebas fueron recogidos a propósito de la investigación, longitudinal(17), lavariable de recirculación del agua residual, en base a sus tres tratamientos, fue realizada en tres fechas distintas, una antes, en el momento y el después, analítico, mediante el análisis estadístico, se planteó la prueba de hipótesis. De nivel descriptivoexplicativo: descriptivo, Se identificó la estrategia necesaria en la recirculación del agua residual industrial en el costo de reúso del proceso, explicativo, confrontar las variables en estudio (Recirculación del Agua Residual Industrial como variable independiente \& Costo del Proceso de Reúso como variable dependiente) (17). Diseño experimental. Con Enfoque Cuantitativo, se determinó a través de la técnica de observación y el instrumento fue el registro del documento de la empresa, para desarrollar el proceso de investigación (18).

En el cercado de la provincia de Pisco, se localizaron los puntos: E-01, E-02 y E-03, considerándose los siguientes parámetros: Porcentaje de reciclo del agua residual de la planta de tratamiento $\left(r_{k}\right)$, concentración de agua de suministros $\left(C_{\text {abas }}\right)$, caudal de entrada al procesos $\left(Q_{e p}\right)$, concentración de entrada de agua al proceso $\left(C_{e p}\right)$, caudal de pérdidas de agua en el proceso $\left(\mathrm{Q}_{\mathrm{pp}}\right)$, carga de contaminantes aportado por el proceso $\left(\mathrm{K}_{\mathrm{pp}}\right)$, carga de contaminantes permitido en la descarga en la fuente $\left(K_{d}\right)$ y costo del agua de suministro $\left(\mathrm{C}_{\text {aguaabas }}\right)$. Se realizó el monitoreo in situ en las fechas coordinadas con la empresa: 14 de octubre, 17 de noviembre y 15 de diciembre del 2020 respectivamente, en los tres puntos seleccionados como se observa en la Figura 1, pruebas realizadas in situ, se accedió a los análisis respectivos para obtener los parámetros del estudio de investigación como se detallan en la Tabla 1 , luego se alimentó al programa denominado curtiembre con extensión Matlab (ANEXO I), y en el programa se desarrolló el algoritmo de costo total de reúso del proceso como lo describe la ecuación 1 y ver la Tabla 2 , en respuesta de la obtención de los datos de costo total se elaboró los niveles y el tratamiento.

Tabla 1. Datos de recirculación del agua residual de la empresa industrial de curtiduría.

\begin{tabular}{llll}
\hline Característica & & \multicolumn{2}{c}{ Datos de Recirculación del agua industrial residual } \\
& 14.10 .2020 & 17.11 .2020 & 15.12 .2020 \\
\hline$r_{k^{*}}$ & 0.60 & 0.675 & 0.75 \\
$\mathrm{C}_{\text {abas }}\left(\mathrm{Kg} / \mathrm{m}^{3}\right)$ & 0.01 & 0.01 & 0.01 \\
$\mathrm{Q}_{\mathrm{ep}}\left(\mathrm{m}^{3} / \mathrm{h}\right)$ & 6.25 & 6.25 & 6.25 \\
$\mathrm{C}_{\mathrm{ep}}\left(\mathrm{Kg} / \mathrm{m}^{3}\right)$ & 0.055 & 0.051 & 0.052 \\
$\mathrm{Q}_{\mathrm{pp}}\left(\mathrm{m}^{3} / \mathrm{h}\right)$ & 2.08 & 2.08 & 2.08 \\
$\left.\mathrm{~K}_{\mathrm{pp}}(\mathrm{Kg}) \mathrm{h}\right)$ & 55.55 & 53.25 & 52.67 \\
$\left.\mathrm{~K}_{\mathrm{pp}}(\mathrm{Kg}) \mathrm{h}\right)$ & 2.6 & 2.65 & 2.62 \\
$\mathrm{C}_{\text {aguabas }}(\mathrm{S} . / \mathrm{h})$ & 2.0 & 2.0 & 2.0 \\
\hline
\end{tabular}

Nota: Dato $\mathrm{r}_{\mathrm{K}}$ : Porcentaje de reciclo del agua residual de la planta de tratamiento. 
Se realizó el monitoreo in situ, siguiendo los criterios establecidos en el protocolo de monitoreo de efluentes líquidos y emisiones atmosféricas, aprobado por Resolución Ministerial N N026-2000-ITINCI-DM protocolo de vigilancia de los recursos hídricos DIGESA N007-VI (20), el reglamento de estándares nacionales de calidad de agua Decreto Supremo No004-2017-MINAN (21) y el Decreto Supremo N003-PRODUCE (22), que refiere sobre límites máximos permisibles y valores referenciales para las actividades industriales de cemento, cerveza, curtiembre y papel, (ANEXO II, Tabla A).

Se utilizó un GPS (Garmin ETREX) para georreferenciar la zona, para levantar información de las coordenadas UTM, sistema: WGS-84, Zona: 18L para la ubicación geográfica de los puntos, además se utilizó un medidor de $\mathrm{pH}$ digital marca OAKTON, Modelo pH 310 para la determinación del potencial de hidrogeno para la determinación de los parámetros químicos de interés, se empleó un equipo MULTIPARAMETRO HACH modelo HQ40d, para la Medición in situ de T, pH, OD y CE. Las unidades que se consideraron para el tratamiento de la recirculación del agua residual industrial en la planta de curtiembre son la poza de sedimentación y el tanque de precipitación, el monitoreo se realizó, según las normas establecidas, (ANEXO II, Tabla B).

Al evaluar, mediante la recirculación del agua residual en el costo de reúso del proceso en la planta curtiduría., se tomó en cuenta desde el suministro del agua al proceso, habiéndose considerado un rango estimado de 0.6 a 0.75 como porcentaje de reciclo del agua residual de la planta de tratamiento, así como también, el porcentaje de carga de retiro en la planta de tratamiento y el porcentaje de carga de retiro en la descarga a la fuente (alcantarillado), como se observa en la Figura 2.

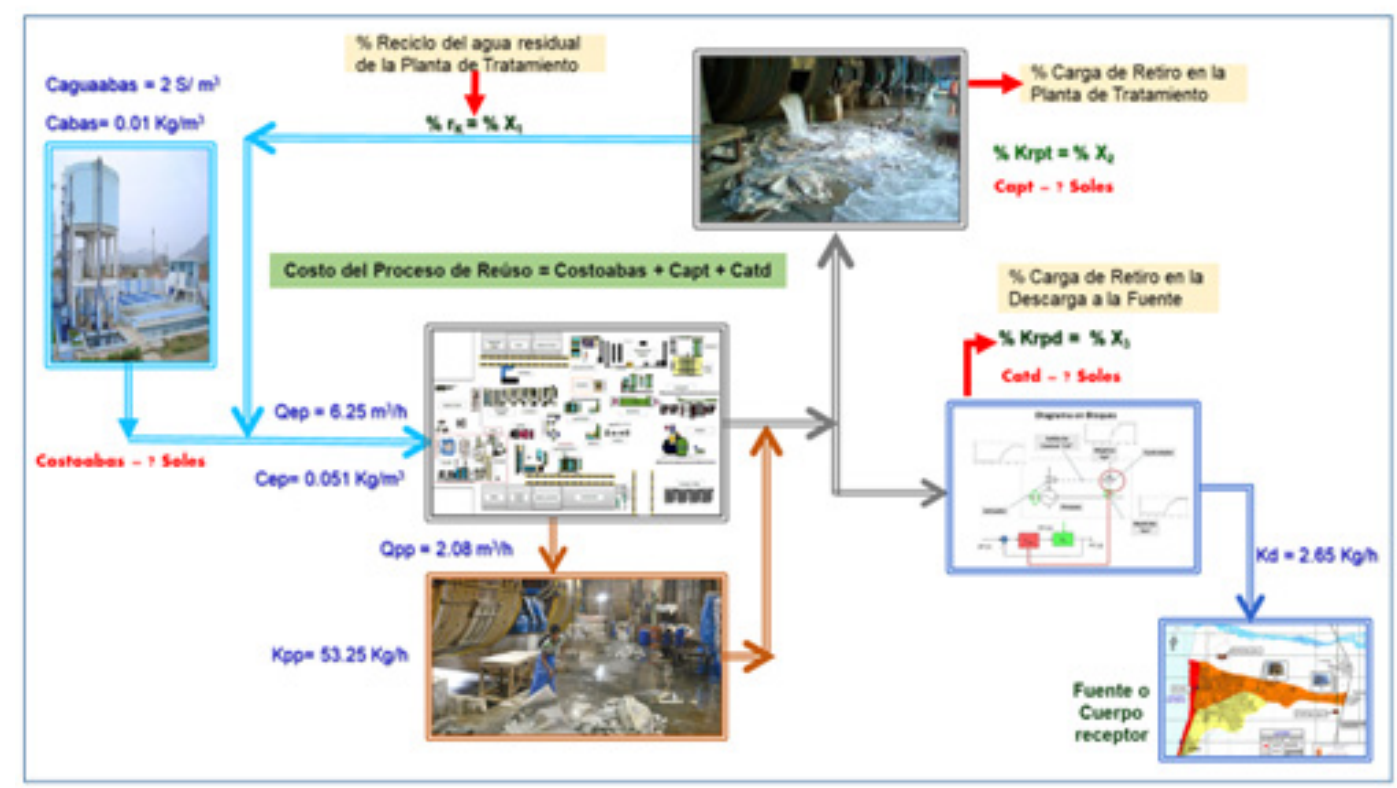

Figura 2. Diagrama de reciclo y la disposición del agua residual industrial tratada. 


\section{Análisis estadístico}

La elección de los niveles y los tratamientos está en base al diseño factorial $2^{3}$, como se señala en la Tabla 2.

Tabla 2. Niveles y Tratamientos.

\begin{tabular}{lccccc}
\hline \multicolumn{1}{c}{ Niveles y Distancia } & Notación & $\mathbf{X}_{\mathbf{1}}(\%)$ & $\mathbf{X}_{\mathbf{2}}(\mathbf{k g} / \mathbf{h})$ & $\mathbf{X}_{\mathbf{3}}(\mathbf{k g} / \mathbf{h})$ & $\mathbf{f}\left(\mathbf{X}_{\mathbf{i}}\right),(\mathbf{S} / \mathbf{d})$ \\
Nivel medio & & 0.675 & 35.86 & 14.76 & 1893.71 \\
Nivel inferior & -1 & 0.60 & 33.22 & 19.72 & 1945.36 \\
Nivel superior & +1 & 0.75 & 39.41 & 10.57 & 1870.98 \\
\hline
\end{tabular}

$\%:$ Reciclo del agua residual de la planta de tratamiento.

$\% \mathrm{Kg} / \mathrm{h}$ : Carga de retiro en la planta de tratamiento y carga de retiro en la descarga a la fuente.

El sistema tal como fue definido, tiene dos variables; [Recirculación del agua residual industrial $(X)$ \& Costo de reúso del proceso $(Y)$ ] $\mathrm{y}$ [tres tratamientos $\left(\mathrm{X}_{1}\right),\left(\mathrm{X}_{2}\right)$ y $\left(\mathrm{X}_{3}\right)$ ], se formuló el modelo general de relación funcional, como se indica en la ecuación 2:

$$
Y=\left(X_{1}, X_{2}, X_{3}, X_{1} X_{2}, X_{1} X_{3}, X_{2} X_{3}, X_{1} X_{2} X_{3}\right)
$$

(Ecuación 2)

Se formularon las siguientes hipótesis estadísticas, Tabla 3.

Tabla 3. La hipótesis estadística para investigar el grado de influencia de los tratamientos en la recirculación en el costo de reúso del proceso.

\begin{tabular}{lll}
\hline HIPOTESIS & $\begin{array}{l}\text { Para la influencia directa de los } \\
\text { tratamientos en la recirculación en el } \\
\text { costo de reúso del proceso }\end{array}$ & $\begin{array}{l}\text { Para la influencia de la interacción de } \\
\text { los tratamientos en la recirculación en } \\
\text { el costo de reúso del proceso }\end{array}$ \\
\hline & $\begin{array}{l}\text { "Los tratamientos directos en la "Los tratamientos de interacción en la } \\
\text { recirculación en el costo de reúso del } \\
\text { proceso no son significativos" }\end{array}$ & $\begin{array}{l}\text { recirculación en el costo de reúso del } \\
\text { proceso no son significativos" }\end{array}$ \\
& $\begin{array}{l}\text { "Los tratamientos directos en la "Los tratamientos de interacción en la } \\
\text { recirculación en el costo de reúso del } \\
\text { proceso son significativos" }\end{array}$ & $\begin{array}{l}\text { recirculación en el costo de reúso del } \\
\text { proceso son significativos" }\end{array}$ \\
\hline
\end{tabular}

$\mathrm{H}_{\mathrm{O}}$ : Hipótesis nula.

$\mathrm{H}_{1}$ : Hipótesis Alternativa. 
RESULTADOS Y DISCUSIÓN a explicar el comportamiento del diseño Utilizando al programa denominado factorial, se realizó la prueba estadística de curtiembre con extensión MATLAB se la varianza de los tratamientos principales obtuvieron números distintos de cero, esto no del diseño básico, $\left(S_{i}^{2}\right)$. El algoritmo de Yates: implica que los tratamientos correspondientes tratamientos y sumas de cuadrados que sean estadísticamente diferentes de cero. Para componen el ANOVA como se muestra en la afirmar que los tratamientos contribuyeron Tabla 5, datos calculados a partir de la Tabla 4.

Tabla 4. Datos obtenidos de la programación con MATLAB, para la elaboración del diseño factorial $2^{3 .}$

\begin{tabular}{|c|c|c|c|c|c|}
\hline $\begin{array}{c}\quad \mathbf{N}^{\circ} \text { de } \\
\text { Combinaciones } \\
\text { Experimentales }\end{array}$ & $\begin{array}{l}\text { Identificación de } \\
\text { La Combinación }\end{array}$ & $X_{1}(\%)$ & $x_{2}(\mathrm{~kg} /)$ & $x_{3}(\mathrm{~kg} / \mathrm{h})$ & $\mathbf{Y}$ \\
\hline 1 & I & 0.60 & 33.22 & 19.72 & 9.70 \\
\hline 2 & $X_{1}$ & 0.75 & 33.22 & 19.72 & 6.40 \\
\hline 3 & $X_{2}$ & 0.60 & 39.41 & 19.72 & 6.20 \\
\hline 4 & $X_{1} X_{2}$ & 0.75 & 39.41 & 19.72 & 1.90 \\
\hline 5 & $X_{3}$ & 0.60 & 33.22 & 10.57 & 5.80 \\
\hline 6 & $X_{1} X_{3}$ & 0.75 & 33.22 & 10.57 & 2.50 \\
\hline 7 & $\mathrm{X}_{2} \mathrm{X}_{3}$ & 0.60 & 39.41 & 10.57 & 3.30 \\
\hline 8 & $\mathrm{X}_{1} \mathrm{X}_{2} \mathrm{X}_{3}$ & 0.75 & 39.41 & 10.57 & 1.30 \\
\hline 9 & & 0.675 & 35.86 & 14.76 & 5.00 \\
\hline 10 & & 0.675 & 35.86 & 19.72 & 5.40 \\
\hline 11 & & 0.675 & 35.86 & 10.57 & 4.20 \\
\hline 12 & & 0.675 & 33.22 & 19.72 & 4.80 \\
\hline 13 & & 0.675 & 39.41 & 19.72 & 4.00 \\
\hline 14 & & 0.60 & 35.86 & 19.72 & 4.60 \\
\hline 15 & & 0.75 & 35.86 & 19.72 & 4.80 \\
\hline
\end{tabular}


Tabla 5. Algoritmo de YATES: efectos y suma de cuadrados, $\mathrm{S}_{i}^{2}$

\begin{tabular}{|c|c|c|c|c|c|c|c|}
\hline $\begin{array}{l}\quad \mathrm{N}^{\circ} \text { de } \\
\text { Combinaciones } \\
\text { Experimentales } \\
\text { Principales } \\
\text { (Diseño Básico) }\end{array}$ & $\begin{array}{c}\text { Identificación de } \\
\text { la Combinación } \\
\text { Clásica }\end{array}$ & $Y(\%)$ & [ 1] & [ 2] & [ 3] & Efectos & $\left(S_{i}^{2}\right)$ \\
\hline 1 & (1) & 9.70 & 16 & 24 & 37 & --- & --- \\
\hline 2 & $X_{1}$ & 6.40 & 8 & 13 & -13 & -3.23 & 1.3001 \\
\hline 3 & $X_{2}$ & 6.20 & 8 & -8 & -12 & -2.93 & 1.0695 \\
\hline 4 & $X_{1} X_{2}$ & 1.90 & 5 & -5 & 0 & 0.07 & 0.0007 \\
\hline 5 & $X_{3}$ & 5.80 & -3 & -8 & -11 & -2.83 & 0.9976 \\
\hline 6 & $X_{1} X_{3}$ & 2.50 & -4 & -4 & 2 & 0.58 & 0.0413 \\
\hline 7 & $X_{2} X_{3}$ & 3.30 & -3 & -1 & 4 & 1.08 & 0.1445 \\
\hline 8 & $X_{1} X_{2} X_{3}$ & 1.30 & -2 & 1 & 2 & 0.58 & 0.0413 \\
\hline
\end{tabular}

$\mathrm{S}_{\mathrm{i}}^{2}=[3]^{2} / 8$

Efectos $=[3] / 4$

$\mathrm{S}_{\mathrm{i}}^{2}:$ Varianza de los tratamientos principales

Tabla 6. Cuadrados de las desviaciones del rendimiento para las combinaciones adicionales.

\begin{tabular}{lllll}
$\begin{array}{c}\mathbf{N}^{\circ} \text { de Combinaciones } \\
\text { Experimentales Adicionales }\end{array}$ & $\mathbf{Y}(\mathbf{S} / \mathbf{d})$ & $(\mathbf{Y}-\mathbf{Y})$ & $(Y-Y)^{2}$ & $S_{\theta}^{2}$ \\
\hline 9 & 5.00 & 0.31 & 0.099 & \\
10 & 5.40 & 0.71 & 0.510 & \\
11 & 4.20 & -0.49 & 0.236 & \\
12 & 4.80 & 0.11 & 0.013 & $S_{\theta}^{2}=\frac{\sum}{(Y-\underline{Y})^{2}}$ \\
13 & 4.00 & -0.69 & 0.470 & $S_{\theta}^{2}=0.225$ \\
14 & 4.60 & -0.09 & 0.007 & \\
15 & 4.80 & 0.11 & 0.013 & \\
$\mathrm{n}=7$ & 32.80 & & 1.349 & \\
& 4.69 & & & \\
\hline
\end{tabular}




\section{Análisis estadístico}

Según el procedimiento, la suma de cuadradosoelcuadradomediodelos resultados para las combinaciones experimentales, se obtuvieron de una forma práctica a través de la varianza de los tratamientos principales del diseño básico, obtenidos por el algoritmo de Yates, $\left(S_{i}^{2}\right)$, Tabla 5.

Para el análisis de varianza (ANOVA), de los resultados obtenidos del diseño factorial se utilizó el F-test $\left(\Phi_{1}, \Phi_{2}\right)$, en base a la varianza de los tratamientos principales por el algoritmo de Yates $\left(S_{i}^{2}\right)$, y la varianza del error experimental e interacción $\left(S_{\theta}^{2}\right)$, ver Tabla 6 . Se evaluó, $\left(S_{\varepsilon}^{2}\right)$, a partir de los resultados de las combinaciones experimentales del 9 al 15 adicionales (Tabla 4), para ello se obtuvo inicialmente el promedio $(\underline{Y}=4.6857)$, luego se construyó la tabla de los cuadrados de las desviaciones de los rendimiontnc nara las combinaciones adicionales $(Y-\underline{Y})^{2}$, y se obtuvo: $\left(S_{e}^{2}=0.2248\right)$. Se efectuó el análisis de varianza con el F-test, comparándose las desviaciones, $\mathrm{S}_{\mathrm{i}}^{2} \mathrm{y} \mathrm{S}_{\mathrm{e}}^{2}$, ver Tabla 7.

Tabla 7. ANOVA y F-test.

\begin{tabular}{lccc}
\hline $\begin{array}{l}\text { Prueba de hipótesis identificación } \\
\text { de la Combinación }\end{array}$ & $\left(S_{i}^{2}\right)$ & $\boldsymbol{\Phi}_{1}$ & $\mathbf{F}\left(\boldsymbol{\Phi}_{1}, \boldsymbol{\Phi}_{2}\right)$ \\
\hline I & --- & --- & - \\
$X_{1}$ & 1.3001 & 1 & 5.7842 \\
$X_{2}$ & 1.0695 & 1 & 4.7582 \\
$X_{1} X_{2}$ & 0.0007 & 1 & 0.0031 \\
$X_{3}$ & 0.9976 & 1 & 4.4384 \\
$X_{1} X_{3}$ & 0.0413 & 1 & 0.1839 \\
$X_{2} X_{3}$ & 0.1445 & 1 & 0.6427 \\
$X_{1} X_{2} X_{3}$ & 0.0413 & 1 & 0.1839 \\
\hline
\end{tabular}

$\Phi_{1}=1$ (Grados de libertad del numerador)

$\Phi_{2}=6$ (Grados de libertad del denominador)

\section{Prueba de Hipótesis}

Los datos obtenidos fueron analizados mediante el $S_{i}^{2}$ y $S_{e}^{2}$. Se procedió a determinar los valores críticos de $F\left(\Phi_{1}, \Phi_{2}\right)$ y $F_{C}\left(\Phi_{1}, \Phi_{2}\right)$, en la (Tabla 8). 
Tabla 8. Cuadro comparativo de $\mathrm{F}\left(\Phi_{1}, \Phi_{2}\right)$, al $95 \%$ de confianza.

\begin{tabular}{|c|c|c|c|c|}
\hline $\begin{array}{c}\text { Grado de confianza } \\
\text { (\%) }\end{array}$ & $F_{c}\left(\Phi_{1}, \Phi_{2}\right)$ & $F\left(\Phi_{1}, \Phi_{2}\right)$ & $\begin{array}{l}\text { Identificación de la } \\
\text { combinación }\end{array}$ & $\begin{array}{l}\text { Grado de influencia del } \\
\text { factor o interacción en la } \\
\text { respuesta }\end{array}$ \\
\hline \multirow{7}{*}{95} & \multirow{7}{*}{5.99} & 5.7842 & $X_{1}$ & Influyente \\
\hline & & 4.7582 & $X_{2}$ & Influyente \\
\hline & & 0.0031 & $X_{1} X_{2}$ & No Influyente \\
\hline & & 4.4384 & $X_{3}$ & Influyente \\
\hline & & 0.1839 & $X_{1} X_{3}$ & No influyente \\
\hline & & 0.6427 & $\mathrm{X}_{2} \mathrm{X}_{3}$ & No influyente \\
\hline & & 0.1839 & $X_{1} X_{2} X_{3}$ & No influyente \\
\hline
\end{tabular}

Se afirma que la recirculación del agua residual industrial al considerar los parámetros del porcentaje de carga de retiro con el porcentaje de reciclo del agua residual $\mathrm{y}$ ambos como $\mathrm{X}_{1} \mathrm{X}_{2}$, esto ha permitido mejorar o incrementar la calidad del agua residual, en el costo de reúso del proceso, procedente de la empresa industrial curtiduría.

Esto quiere decir que los resultados de la prueba de hipótesis al $95 \%$ de confianza, se concluye que el costo de reúso del proceso depende de la siguiente relación funcional de $\mathbf{f}\left(\mathbf{X}_{1}, \mathbf{X}_{2}, \mathbf{X}_{1} \mathbf{X}_{2}\right)$, donde las combinaciones de $\left(X_{1}=5.7842, X_{2}=4.7582\right.$ y $\left.X_{1} X_{2}=4.4384\right)$, frente $a$ lo mencionado se rechaza la hipótesis nula $(\mathrm{Ho})$ y se acepta la hipótesis alternativa $(\mathrm{Ha})$.

Estos resultados son corroborados por quienes en su investigación concluyen que las funciones de respuesta para el porcentaje de mineralización desarrollados en las ecuaciones de reacciones de Fenton y fotoFenton (23), donde aplicaron la prueba de
Yates y el análisis de varianza (ANOVA), siendo significativos de $95 \%$ (valor con un margen de error de +/- 0.05), para ello, se realizaron estudios en una simulación de aguas residuales agroindustriales, se utilizó el diseño estadístico de experimentos para evaluar la influencia de la concentración de IMZ, Fe (II) y $\mathrm{H}_{2} \mathrm{O}_{2}$ en la eficiencia del proceso, el Fe (II) y $\mathrm{H}_{2} \mathrm{O}_{2}$ optimizados para la mineralización de $50 \mathrm{mg} \mathrm{L}^{-1}$ IMZ fueron 21.5 y $127.5 \mathrm{mg} \mathrm{L}^{-1}$, respectivamente, se estimó un costo operativo de $2.06 \mathrm{~s} / \mathrm{m}^{3}$ para el tratamiento de $10 \mathrm{~m}^{3}$ de aguas residuales contaminadas con IMZ, esto nos permite argumentar con mayor criterio que el diseño factorial $2^{3}$ es una técnica estadística que permite desarrollar los efectos o tratamientos de la variable independiente. Así también, se refieren que el análisis de datos estadísticos de los resultados del diseño factorial (24), se estimó el análisis de varianza (ANOVA) que le permitió determinar la significancia entre $y$ dentro de los niveles bajo y alto de cada factor 
en $\alpha=0.05$, con el fin de determinar todos los términos viables, siendo su aporte de los investigadores sobre los efectos principales y las de interacción, como el gráfico de Pareto, además considera el gráfico de probabilidad normal. Permitiendo en futuras investigaciones considerar como factores o variables de entrada: mediciones, variaciones, efectos directos y efectos de iteración en procesos fisicoquímicos, biológicos y sanitarios.

En tal sentido, en base a lo referido anteriormente y al analizar estos resultados confirmamos que la carga de retiro en la descarga a la fuente o cuerpo receptor $\left(\mathrm{X}_{3}\right)$, como el efluente crudo de la curtiembre, no genera impacto de contaminación alguno al ser lanzados a una red de alcantarillado. Se menciona que la recirculación de las aguas residuales industriales (14), es un tema importante que debe ser considerado también en los países latinoamericanos, como en nuestra realidad nacional, considerando la realidad plantea de los 17 Objetivos de Desarrollo Sostenible (ODS) definidos por Naciones Unidas que abordan los mayores retos de la humanidad desde una perspectiva global y transversal, con una visión a futuro hacia el año 2030. Es importante fortalecer el objetivo 6 de la ODS, de la calidad de agua y saneamiento, sobre la escasez de agua, los problemas de calidad del agua y el saneamiento inadecuado trabajando por un uso racional y sostenible de este recurso de primera necesidad y afrontando los riesgos relacionados con su escasez.

\section{CONCLUSIONES}

El propósito de este artículo fue analizar la recirculación del agua residual industrial en el costo de reúso del proceso, con los datos obtenidos con un nivel de significancia de $95 \%$ con un margen de error de +/-0.05, se determinó el grado de influencia de los tratamientos en la recirculación en el costo de reúso del proceso $X_{1}, X_{2}, X_{1} X_{2}$. De tal manera que (S/d) de 1945.36 a 1870.98, que depende del porcentaje de reciclo del agua residual de la planta de tratamiento de 0.6 a 0.75 , con el porcentaje de retiro en la planta de tratamiento de 33.22 $\mathrm{Kg} / \mathrm{h}$ a $39.41 \mathrm{Kg} / \mathrm{h}$ y de ambos del porcentaje de reciclo del agua residual como del porcentaje de retiro en la planta de tratamiento. Es así que en los tratamientos del agua residual permitió tomar en cuenta la aplicación de tecnologías conocidas para mejorar o incrementar la calidad del agua residual, es por ello que los procesos físicos, químicos y fisicoquímicos juegan un papel importante en el tratamiento del agua residual en la empresa industrial de curtiduría.

Se recomienda que las futuras investigaciones en la determinación de la influencia en el costo del reusó de las aguas residuales industriales tengan en consideración los parámetros de carga contaminante específica.

\section{REFERENCIAS BIBLIOGRÁFICAS}

1. Tamayo SS, Esquivel EM. El desarrollo Industrial y su Impacto en el Medio Ambiente. Rev cubana Hig Epidemiol. 2014;52(3):357-363. http://scielo.sld.cu/pdf/hie/v52n3/hig08314. pdf 
2. Navarro T. Water reuse and desalination in Spain - challenges and opportunities. J Water Reuse Desalin. 2018;8(2):153-168 pag. doi:https://doi.org/10.2166/wrd.2018.043

3. Sandin G, Peters GM. Environmental impact of textile reuse and recycling - A review. J Clean Prod. 2018; 184:353-365. doi: 10.1016/j. jclepro.2018.02.266

4. Kant R. Textile dyeing industry an environmental hazard. Nat Sci. 2012;4(1):22-26 pag. doi:10.4236/ns.2012.41004

5. Hussain T, Wahab A. A critical review of the current water conservation practices in textile wet processing. J Clean Prod. 2018; 198:806819. doi: 10.1016/j.jclepro.2018.07.051

6. Arora S. Textile Dyes: It's Impact on Environment and its Treatment. J Bioremediation Biodegrad. 2014;05(03):6199. doi:10.4172/2155-6199.1000e146

7. Holkar CR, Jadhav AJ, Pinjari D V., Mahamuni NM, Pandit AB. A critical review on textile wastewater treatments: Possible approaches. J Environ Manage. 2016; 182:351-366. doi: 10.1016/j.jenvman.2016.07.090

8. $\mathrm{N}^{\circ} 29338 \mathrm{~L}$ de RH. Ley de Recursos Hídricos $\mathrm{N}^{\circ}$ 29338. Published online 2009:40 pp. http://www.minam.gob.pe/wp-content/ uploads/2017/04/Ley- $\mathrm{N}^{\circ}$-29338.pdf

9.Del Castillo GutiérrezM, Meseth Macchiavello E. El Reuso De Aguas Residuales Tratadas En El Perú. ResearchGate. 2015;(December):5

10. Marangon BB, Silva TA, Calijuri ML, Alves $S$ do $C$, dos Santos VJ, Oliveira AP de S. Reuse of treated municipal wastewater in productive activities in Brazil's semi-arid regions. J Water Process Eng. 2020;37(May):101483. doi: 10.1016/j.jwpe.2020.101483

11. Olivieri AW, Pecson B, Crook J, Hultquist R. California water reuse-Past, present and future perspectives. Vol 5. 1. ${ }^{a}$ ed. Elsevier Inc.; 2020. doi: 10.1016/bs.apmp.2020.07.002

12. Fuente $D$. The design and evaluation of water tariffs: A systematic review. Util Policy. 2019;61(September):100975. doi: 10.1016/j. jup.2019.100975
13. Martinez S, Rodriguez M. Tratamiento de aguas residuales con matla. Reverte Ediciones S.A. de C.V.; 2005

14. Naghedi R, Alavi Moghaddam MR, Piadeh F. Creating functional group alternatives in integrated industrial wastewater recycling system: A case study of Toos Industrial Park (Iran). J Clean Prod. 2020; 257:120464. doi: 10.1016/j.jclepro.2020.120464

15. Ayesa Iturrate $E$, Larrea Urcola MA. Optimización del diseño, operación y control de las EDAR utilizando modelos matemáticos y herramientas de simulación. Tecnol del Agua. 2005;25(266):74-79

16.INEI.INSTITUTONACIONALDEESTADÍSTICA E INFORMÁTICA.; 2017. https://www.inei.gob. pe/media/MenuRecursivo/publicaciones_ digitales/Est/Lib1496/libro.pdf

17. Supo J. Cómo escribir una tesis: Redacción del informe final de tesis. Primera Ed. BIOESTADISTICO EIRL; 2015

18. Carrasco Diaz S. METOdOLOGÍA DE LA INVESTIGACIÓN CIENTÍFICA METODOLOGÍA DE LA INVESTIGACIÓN CIENTÍFICA. Pautas metodológicas para diseñar y elaborar el proyecto de investigación. (LTDA ESMEIR, ed.).; 2019. http://www.sancristoballibros. com/libro/metodologia-de-la-investigacioncientifica_45761

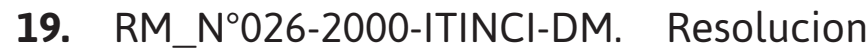
Ministerial N026-2000-ITINCI-DM. Published online 2002:38 pag. https://www. legislacionambientalspda.org.pe/images/ stories/normas/Pioner 2/IV. 4. Industrial/4. Resoluciones ministeriales/Resolucion ministerial 026-2000-ITINCI-DM.doc

20. DIGESA_007_VI. Procedimientos Para la Vigilancia de los Recursos HidricosICOS - Seguimiento de la(s) Muestra(s) Published online 2002:6 pag. https://www. legislacionambientalspda.org.pe/images/ stories/normas/Pioner 2/IV. 4. Industrial/4. Resoluciones ministeriales/Resolucion ministerial 026-2000-ITINCI-DM.doc 
21. Decreto_Supremo_No-004-MINAM. Estándares de Calidad Ambiental para Agua (ECA).; 2017. http://www.minam.gob.pe/ wp-content/uploads/2017/06/DS-004-2017MINAM.pdf

22. DS_N003-2008-MINAM. Aprueban Estnadares de Calidad Ambiental Para Aire. Decreto Supremo N003-2008-MINAM. D Of El Peru. Published online 2008:4 Pag

23. Santiago DE, González-Díaz O, Araña J, Pulido Melián E, Pérez-Peña J, Doña-Rodríguez JM. Factorial Experimental Design of Imazalil-
Containing Wastewater to be Treated by Fenton-Based Processes. J Photochem Photobiol A Chem. 2018; 353:240-250 pag. doi: 10.1016/j.jphotochem.2017.11.038

24. Moghazy RM, Labena A, Husien S. Ecofriendly Complementary Biosorption Process of Methylene Blue Using Micro-sized Dried Biosorbents of two Macro-Algal Species (Ulva fasciata and Sargassum dentifolium): Full Factorial Design, Equilibrium, and Kinetic Studies. Int J Biol Macromol. 2019; 134:330-343. doi: 10.1016/j.ijbiomac.2019.04.207 
ANEXO I (13)

clc

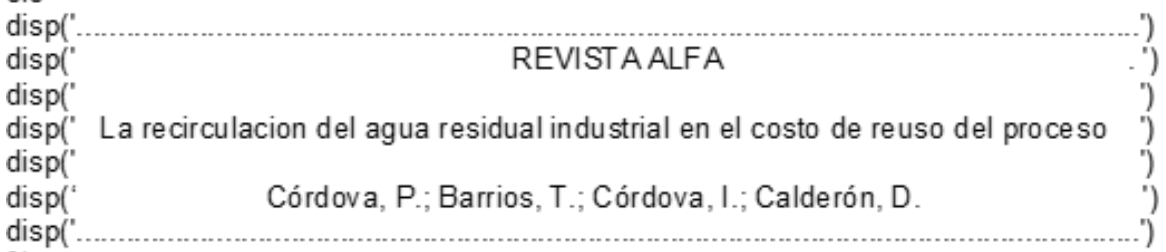

$\%$

$\%$ Programa curtiduria

$\%$

Cabas = input('Concentracion de agua de abastecimiento $\left(\mathrm{kg} / \mathrm{m}^{\wedge} 3\right)$ : ");

Qep = in put('Caudal de entrada al proceso $\left(\mathrm{m}^{\wedge} 3 / \mathrm{h}\right)$ : ');

Cep = input('Concentracion permitida en agua de proceso $\left(\mathrm{kg} / \mathrm{m}^{\wedge} 3\right)$ : ");

$Q p p=$ in put('Caudal de perdida de agua $\left(\mathrm{m}^{\wedge} 3 \mathrm{~h}\right)$ : " );

$\mathrm{Kpp}=$ input('Carga aportada por el proceso $(\mathrm{kg} / \mathrm{h}):$ :);

$\mathrm{Kd}=$ in put('Carga permitidos en la de scarga $(\mathrm{kg} / \mathrm{h}):$ : ");

Caguaabas $=$ in put('Costo del agua de abastecimiento $\left(S / m^{\wedge} 3\right)$ : ');

$\%$

for rec $=0.0: .05: 0.90$

format short $\mathrm{g}$

$\mathrm{Q} r=\mathrm{rec}{ }^{\star} \mathrm{Q}$ ep; \%Flujo Agua de reciclo

Qabas $=Q$ ep-Qr \%Flujo de agua proveniente del abastecimiento

$\mathrm{Qsp}=\mathrm{Q}$ ep-Qpp; \%Flujo de agua proveniente del proce so

Qat $=\mathrm{Qsp}+\mathrm{Qpp}$; \%Flujo de agua del proceso más la perdida de agua

Qapt $=\mathrm{Qr} ; \%$ Flujo de agua para tratamiento y reciclo

Qfuente=Qat-Qapt; \%Flujo de agua de de scarga en el cuerpo receptor

Kabas $=Q$ abas ${ }^{*}$ Cabas; \%Carga en el agua de abastecimiento

Kep $=Q$ ep ${ }^{\star} \mathrm{Cep}$; \%Carga en el agua de entrada al proceso

$\mathrm{Kr}=\mathrm{Kep}-\mathrm{Kabas} ; \%$ Carga del agua proveniente de la planta de tratamiento

Ksp=Kep; \% Carga de contaminantes a la salida del proceso

$\mathrm{Kat}=\mathrm{Kpp}+\mathrm{Ksp}$; \%Carga en el agua residual para tratamiento

Kapt=Kat ${ }^{\star}(\mathrm{Q} a p t / Q$ at); $\%$ Carga en el reciclo entrada a tratamiento

Kapd=Kat-Kapt; \%Carga en el caudal para el tratamiento y descarga

Krpt=Kapt-Kr;\%Carga de contaminantes removida del agua de reú so

ER1 $=100 *$ Krpt $/$ Kapt;\%Porcentaje

if $E R 1<=0$

$\mathrm{ER}=0$;

else

$\mathrm{ER}=\mathrm{ER} 1$;

end

$\mathrm{Krpd}=\mathrm{Kapd}-\mathrm{Kd} ; \%$ Carga removida del caudal para descarga a la fuente

$\mathrm{ED}=100^{*} \mathrm{Krpd} / \mathrm{Kapd} ; \%$ porcentaje

$x=0.0012 ; y=0.0127 ; z=0.0047$;

$A=\left(\left(x^{\star} E R^{\wedge} 2\right)+y^{\star} E R+z\right)$;

$\mathrm{B}=\left(\left(\mathrm{x}^{*} E D^{\wedge} 2\right)+\mathrm{y}^{\star} \mathrm{ED}+\mathrm{z}\right)$

Capt $=$ Qapt ${ }^{\star} 24{ }^{*} \mathrm{~A} ; \%$ C osto re ciclo

Capdr $r=$ Qfuente ${ }^{\star} 24^{*} B ; \%$ Costo disposicion

if $\mathrm{Capdr}<0 \%$ no requiere tratamiento para descarga al cuerpo receptor

Capd $=0$;

else

Capd=Capdr;

end

Costo abas $=\left(\right.$ 'C aguaabas ${ }^{\star}{ }^{2}$ abas ${ }^{\star} 24$ '); \%costo abastecimiento

Costototal $=($ Capt + Capdr + Costo abas $) ; \%$ co sto total

xlabel('rec');

ylabel('Costototal (S/d)');

plot(rec,Costototal,'k+')

grid

hold on

disp(" rec Costototal (S/d)")

$\mathrm{R}=[\mathrm{rec}$, Costototal]

end 


\section{ANEXO II}

El efluente fue tomado en el interior de la planta industrial, previa descarga (salida de la planta de tratamiento) a la red pública de Emapisco.Elanálisiscomprendiólosparámetros contemplados en el D.S. 003-2002-PRODUCE, “Límites Máximos Permisibles de los Efluentes para Alcantarillado de las actividades de Curtiembre". Los resultados se muestran en las siguientes tablas.

Tabla A. Límite máximo permisible de efluentes para alcantarillado de las actividades de cemento, cerveza, papel y curtiembre.

\begin{tabular}{|c|c|c|c|c|c|c|c|c|}
\hline \multirow[b]{2}{*}{ Parámetros } & \multicolumn{2}{|c|}{ Cemento } & \multicolumn{2}{|c|}{ Cerveza } & \multicolumn{2}{|c|}{ Papel } & \multicolumn{2}{|c|}{ Curtiembre } \\
\hline & En Curso & Nueva & En Curso & Nueva & $\begin{array}{c}\text { En } \\
\text { Curso }\end{array}$ & Nueva & $\begin{array}{c}\text { En } \\
\text { Curso }\end{array}$ & Nueva \\
\hline $\mathrm{PH}$ & $6-9$ & $6-9$ & $6-9$ & $6-9$ & $6-9$ & $6-9$ & & $6-9$ \\
\hline Temperatura $\left({ }^{\circ} \mathrm{C}\right)$ & 35 & 35 & 35 & 35 & 35 & 35 & 35 & 35 \\
\hline Solidos Susp Tot(mg/l) & 100 & 50 & 500 & 350 & 1000 & 500 & & 500 \\
\hline Aceites y Grasas (mg/l) & & & 20 & 15 & 100 & 50 & 100 & 50 \\
\hline DBO5 (mg/l) & & & 1000 & 500 & & 500 & & 500 \\
\hline $\mathrm{DQO}(\mathrm{mg} / \mathrm{l})$ & & & 1500 & 1000 & & 1000 & & 1500 \\
\hline Sulfuros (mg/l) & & & & & & & & 3 \\
\hline Cromo VI (mg/l) & & & & & & & & 0,4 \\
\hline Cromo total (mg/l) & & & & & & & & 2 \\
\hline $\mathrm{N}-\mathrm{NH} 4(\mathrm{mg} / \mathrm{l})$ & & & & & & & & 30 \\
\hline Coliformes Fecales NMP/100ml & & & & & & & * & * \\
\hline
\end{tabular}

* En el caso del Subsector Curtiembre, no se ha fijado valores para el parámetro Coliformes fecales, dado que la data recopilada no era representativa, ni confiable. Asimismo, no ha sido posible identificar data a nivel nacional, ni en los países analizados sobre LMP específicos para este parámetro en curtiembres.

Tabla B. Resultado de monitoreo efluente.

\begin{tabular}{|c|c|c|c|}
\hline Parámetro & $\begin{array}{c}E F-03 \\
(N: 8483762, E: 371843)\end{array}$ & LMP & Unidad \\
\hline $\mathrm{pH}$ & 7,69 & $6-9$ & Unidad pH \\
\hline Temperatura & 30,0 & 35 & ${ }^{\circ} \mathrm{C}$ \\
\hline Sólidos Suspendidos Totales & 71,86 & 500 & $\mathrm{mg} / \mathrm{L}$ \\
\hline Aceites y Grasas & 16,1 & 50 & $\mathrm{mg} / \mathrm{L}$ \\
\hline Demanda Bioquímica de Oxígeno $\left(\mathrm{DBO}_{5}\right)$ & 369,50 & 500 & $\mathrm{mg} / \mathrm{L}$ \\
\hline Demanda Química de Oxígeno (DQO) & 1432,0 & 1500 & $\mathrm{mg} / \mathrm{L}$ \\
\hline Sulfuros & 31 & 3 & $\mathrm{mg} / \mathrm{L}$ \\
\hline Cromo VI & $<0,007$ & 0,4 & $\mathrm{mg} / \mathrm{L}$ \\
\hline Cromo total & 0,0004 & 2 & $\mathrm{mg} / \mathrm{L}$ \\
\hline $\mathrm{N}-\mathrm{NH}_{4}$ & 27,1 & 30 & $\mathrm{mg} / \mathrm{L}$ \\
\hline
\end{tabular}

